\title{
Genetic map of regional sulcal morphology in the human brain
}

Benjamin B. Sun ${ }^{1,2}$, Stephanie J. Loomis ${ }^{1 \uparrow}$, Fabrizio Pizzagalli ${ }^{3,4 \uparrow}$, Natalia Shatokhina ${ }^{4}$, Jodie N. Painter ${ }^{5}$, Christopher N. Foley ${ }^{6,7}$, Biogen Biobank Team ${ }^{1}$, Megan E. Jensen ${ }^{8}$, Donald G. McLaren $^{8}$, Sai Spandana Chintapalli ${ }^{9}$, Alyssa H. Zhu ${ }^{4}$, Daniel Dixon ${ }^{4}$, Tasfiya Islam ${ }^{4}$, Iyad Ba Gari $^{4}$, Heiko Runz ${ }^{1}$, Sarah E. Medland ${ }^{5}$, Paul M. Thompson ${ }^{4 *}$, Neda Jahanshad ${ }^{4 *}$, Christopher D. Whelan ${ }^{1 *}$

1. Translational Biology, Research \& Development, Biogen Inc., Cambridge, MA, US

2. BHF Cardiovascular Epidemiology Unit, Department of Public Health and Primary Care, University of Cambridge, Cambridge, UK

3. Department of Neuroscience "Rita Levi Montalcini", University of Turin, Turin, Italy

4. Imaging Genetics Center, Mark and Mary Stevens Neuroimaging and Informatics Institute, Keck School of Medicine, University of Southern California, Marina del Rey, CA, US

5. QIMR Berghofer Medical Research Institute, Brisbane, Queensland, Australia

6. MRC Biostatistics Unit, School of Clinical Medicine, University of Cambridge, Cambridge, UK

7. Optima Partners, Edinburgh, UK

8. Clinical Sciences, Research \& Development, Biogen Inc., Cambridge, MA, US

9. Department of Bioengineering, University of Pennsylvania, Philadelphia, PA, US

$\dagger$ Authors contributed equally

* Authors contributed equally 


\section{Abstract}

26 The human brain is a complex organ underlying many cognitive and physiological processes,

27 affected by a wide range of diseases. Genetic associations with macroscopic brain structure are 28 emerging, providing insights into genetic sources of brain variability and risk for functional

29 impairments and disease. However, specific associations with measures of local brain folding,

30 associated with both brain development and decline, remain under-explored. Here we carried

31 out detailed large-scale genome-wide associations of regional brain cortical sulcal measures

32 derived from magnetic resonance imaging data of 40,169 individuals in the UK Biobank.

33 Combining both genotyping and whole-exome sequencing data ( $\sim 12$ million variants), we

34 discovered 388 regional brain folding associations across 77 genetic loci at $p<5 \times 10^{-8}$, which

35 replicated at $p<0.05$. We found genes in associated loci to be independently enriched for

36 expression in the cerebral cortex, neuronal development processes and differential regulation

37 in early brain development. We integrated coding associations and brain eQTLs to refine genes

38 for various loci and demonstrated shared signal in the pleiotropic KCNK2 locus with a cortex-

39 specific KCNK2 eQTL. Genetic correlations with neuropsychiatric conditions highlighted

40 emerging patterns across distinct sulcal parameters and related phenotypes. We provide an

41 interactive 3D visualisation of our summary associations, making complex association patterns

42 easier to interpret, and emphasising the added resolution of regional brain analyses compared

43 to global brain measures. Our results offer new insights into the genetic architecture

44 underpinning brain folding and provide a resource to the wider scientific community for studies

45 of pathways driving brain folding and their role in health and disease.

46 


\section{Main}

48 Human brain structure and function are complex drivers of basic and higher cognitive

49 processes, which vary between individuals and in numerous neurological, psychiatric and

50 cognitive disorders. Structural magnetic resonance imaging (MRI) scans provide a reliable, non-invasive measure of brain structure and are widely used in research and clinical settings.

52 Genetic variants influencing brain structure and function are important to identify, as they can

53 help uncover pathophysiological pathways involved in heritable brain diseases and prioritize

54 novel targets for drug development. Several large-scale genome-wide association studies

55 (GWAS) have identified hundreds of genetic influences on variations in brain structure and function ${ }^{1-3}$ revealing novel insights into processes guiding brain development, and highlighting potential shared genetic aetiologies with neurodegenerative and psychiatric conditions ${ }^{4,5}$.

59 To date, most neuroimaging GWAS have focused on broad, macroscale anatomical features

60 such as subcortical volume, cortical thickness and white matter microstructure ${ }^{6}$. Anomalies of cortical gyrification - the folding of the cerebral cortex into its characteristic concave sulci (fissures) and convex gyri (ridges) - contribute to many neurodevelopmental and neuropsychiatric conditions ${ }^{7,8}$, but the genetic underpinnings of gyrification remain relatively understudied ${ }^{9}$. Sulcal characteristics and folding patterns are altered across a range of neurodevelopmental disorders, from cortical dysplasias ${ }^{10}$ to neurogenetic syndromes ${ }^{11}$, and radiologists often use sulcal widening as an early indicator of atrophy in degenerative

67 diseases $^{12}$, as it offers a clear and sensitive biomarker of disease progression ${ }^{13,14}$. Recent neuroimaging genetics investigations have broadened in scale and scope, examining specific

69 sulcal measures across the full brain ${ }^{15,16}$, but without evaluating the reliability of the measures at scale across MRI scanning protocols. Using four independent datasets, we recently outlined 
71 a range of heritable sulcal measures that can be reliably quantified at high resolution across the

72 whole brain, irrespective of MRI platform or acquisition parameters ${ }^{17}$.

74 Here we conducted a comprehensive genome-wide analysis of regional sulcal shape 75 parameters, extracted from the multi-centre brain MRI scans of 40,169 participants in the UK

76 Biobank. To discover rare and common genetic variants influencing cortical gyrification, we conducted GWAS and exome-wide analysis of a total of 450 sulcal parameters ${ }^{17}$. Sulcal shape

78 descriptors, comprising length, mean depth, width, and surface area, were extracted from a 79 discovery cohort of 26,530 individuals of European ancestry and a replication cohort of 13,639

80 individuals. After mapping the genetic architecture of regional sulcal measures across the 81 cortex, we highlight putative biological and developmental pathway involvement as well as 82 links to neuropsychiatric conditions. Finally, we provide a portal to interactively visualise our 83 results in 3D (https://enigma-brain.org/sulci-browser), demonstrating varying complex 84 patterns of associations, to help inform future investigations of human cortical morphology. 


\section{Results}

87 Regional brain sulcal measurements (4 shape parameters: length, width, mean depth and 88 surface area), regional delineations, and phenotype nomenclature are summarised in

89 Supplementary Table 1 and Figure 1a. We determined the overall clustering of the high-

90 dimensional phenotypes with $t$-SNE and found that width parameter phenotypes formed a

91 distinct cluster compared to the other three shape parameters (Extended Data Figure 1).

92 Notably, the t-SNE representation retains broad brain lobe topology for the width parameter

93 phenotypes in particular (Figure 1b).

\section{Genetic architecture of regional brain sulcal folds}

We conducted GWAS of 450 regional brain sulcal measurements separately for 11.9 million combined imputed and whole-exome sequenced variants in UKB participants divided into a discovery cohort $(n=26,530)$ and a replication cohort $(n=13,639)$ (Methods, Extended Data

\section{Figure 2).}

At a significance threshold of $p<2 \times 10^{-10}$ which accounts for the effective number of independent sulcal measures analysed (Methods) - we found, and replicated at $p<0.05$, a total of 186 specific sulci parameter associations (for at least one hemisphere) across 41 genetic loci (388 associations across 77 loci at $p<5 \times 10^{-8}$ ) (Figure 1c and 1d, Extended Data Figure 3, Supplementary Table 2). We also performed GWAS on bilateral sulcal measurements (averaging values from left and right brain hemispheres) and found a total of 162 replicated associations across 47 loci at $p<2 \times 10^{-10}$ (335 associations across 107 loci at $p<5 \times 10^{-8}$ ), where 6 (across 3 loci) and 108 additional associations (across 42 loci) were also found at $p<2 \times 10^{-10}$ and $p<5 \times 10^{-8}$ respectively (Supplementary Table 3). Genomic inflation was well controlled (median $\lambda \mathrm{gc}=1.02$, range: $0.99-1.07$ ). We found an inverse relationship between effect sizes 
111 and minor allele frequency (MAF) (Extended Data Figure 4), in line with other disease and

112 intermediate trait results, and consistent with variants with strong effects are deleterious and

113 rarer.

115 We found a similar number of associations for left and right hemispheres. Approximately two-

116 thirds of associations were with sulcal width, followed by mean depth, surface area and length

117 measures, in line with their heritability estimates ${ }^{17}$ (Figure 1c and 1d, Extended Data Figure

118 5). Length measures accounted for the lowest proportion of associations $(<5 \%$, Figure 1b),

119 consistent with length having the lowest heritability (Extended Data Figure 5), especially

120 after adjusting for intra-cranial volume ${ }^{17}$. Comparing the absolute (all effects across

121 hemispheres were consistent in direction) Z-scores of lead associations across hemispheres

122 (left, right and bilateral, Extended Data Figure 6), we found no significant difference between

123 left and right hemisphere (paired t-test $p=0.25$ ), whilst bilateral associations tended to exhibit

124 stronger associations (mean abs(Z-score) 1.00 higher $v s$ right, $p=4.1 \times 10^{-96}$ and 0.92 higher $v s$

125 left, $p=1.8 \times 10^{-82}$ ), consistent with their heritability estimates (Extended Data Figure 5 and 6 ).

127 Some genetic loci exhibited highly pleiotropic associations across multiple brain regions; for

128 example, 10 genetic loci were associated with 10 or more sulcal measures, showing different

129 association patterns across shape parameters. Notably, the chr1:215Mb (near KCNK2) and

130 chr12:106Mb (12q23.3, NUAK1) regions were associated with 23 and 22 width measures

131 respectively across multiple brain regions; the chr16:87Mb region (6q24.2, near C16orf95) was

132 associated with 16 width measures across multiple brain regions, 4 mean depth and 1 surface

133 area measures mostly in the frontal lobe; the chr17:47Mb region (17q21.31, containing $M A P T$

134 and KANSL1) was associated with 16 width, 9 surface area, 6 mean depth and 2 length

135 measures mostly in the temporal and calcarine-occipital regions; whilst chr6:126Mb region 
136 (6q22.32, containing $C E N P W)$ was associated with 9 surface area, 4 length, 4 mean depth and

1372 width measures - mostly in the frontal and calcarine-occipital regions (Figure 1c,

138 Supplementary Table 2).

140 We cross-referenced the lead variants and their proxies $\left(\mathrm{r}^{2}>0.8\right)$ for significant $\left(p<5 \times 10^{-8}\right)$ associations in previous related brain imaging studies (Supplementary Information) in the GWAS Catalog ${ }^{6}$ (LD proxy $\mathrm{r}^{2}>0.8,+/-500 \mathrm{~Kb}$ around the lead variant). We found 56 of the 119 loci (77 for left/right hemisphere and 42 bilateral measures, $p<5 \times 10^{-8}$ ) to be associated with any brain imaging phenotype (mostly consisting of brain volume, surface area and white matter microstructure) including the 10 highly pleiotropic genetic loci, many of which (e.g. CENPW containing locus (6q22.32), MAPT-KANSL1 containing locus (17q21.31), C16orf95 locus (6q24.2), NUAK1 locus (12q23.3), chr2:65Mb (2p14), chr15:40Mb (15q14), chr14:59Mb (14q23.1) loci) were previously implicated across multiple studies (Supplementary Table 4).

Over half of our regional brain sulcal associations identified were not previously implicated in any published brain imaging related studies.

\section{Coding variant associations}

153 We also examined whether any of the lead variants were in strong $\operatorname{LD}\left(\mathrm{r}^{2}>0.8\right)$ with coding

154 variants $\left(p_{\text {discovery }}<5 \times 10^{-8}\right.$ and $\left.p_{\text {replication }}<0.05\right)$. We identified 10 loci harbouring coding variants

155 or proxies (coding/splice region variants) in strong LD with lead variants (Supplementary

156 Table 5). With the exception of the complex chr17:47Mb (17q21.31, MAPT) locus, which 157 contained coding/splice region proxies for multiple genes (ARHGAP27, PLEKHM1, CRHR1, 158 SPPL2C, MAPT, STH, KANSL1), the other 9 loci contained coding variants affecting proxies 159 for single genes (ROR1 [rs7527017, Thr518Met], THBS3 [rs35154152, Ser279Gly], SLC6A20 160 [rs17279437, Thr199Met], EPHA3 [rs35124509, Trp924Arg], MSH3 [rs1650697, Ile79Val], 
GNA12 [rs798488, start-lost], PDGFRL [rs2705051, splice region variant], EML1 [rs34198557, indel with stop codon gained]). Notably, the SLC6A20 Thr199Met (rs17279437) variant, associated with widespread reductions in sulcal width (Supplementary Table 5), has previously been associated with reduced thickness of retinal components and with increased glycine and proline derivatives in CSF and urine, consistent with the role of SLC6A20 as cotransporter regulating glycine and proline levels in the brain and kidneys, highlighting proline/glycine pathways in regulating brain sulcal widths (see Supplementary Information

169 for details).

\section{Genetic and phenotypic correlations of brain folding}

172 We investigated the phenotypic and genetic correlation (GC) between measures from the right and left hemispheres as well as between different shape parameters of the brain sulcal measurements. We found high correlations between brain sulcal measurements across left and right sides, within and between the four shape parameters (Figure 1c, Extended Data Figure 7). In general, the strongest correlations were detected between left and right hemispheres for width compared to length, mean depth and surface area (Figure 1c top). The high genetic correlation between hemispheres may explain the higher magnitudes of the association Zscores of bilateral brain sulcal measures compared to hemisphere-specific analyses. We found

180 average length, mean depth and surface area parameters to be positively correlated, with correlation between length and surface area the strongest, and width to be negatively correlated 
187 Brain folding genes enriched for cortical expression and

\section{8 neurodevelopmental processes}

189 To determine whether genes in the associated regions were enriched for expression in certain

190 tissues, we performed enrichment analysis of annotated genes in significant loci $\left(p<5 \times 10^{-8}\right)$ for

191 tissue gene expression in an independent dataset (Human Protein Atlas) (Methods). We found significant enrichment of brain folding genes of approximately two-fold for expression in the cerebral cortex after multiple testing correction $\left(p=7.3 \times 10^{-7}\right)$. This effect remained significant with other sensitivity analysis thresholds (Figure 2a), suggesting associated brain folding genes may have local effects. We also performed enrichment analysis for gene ontology (GO) processes and KEGG pathways. Notably, we found significant $(\mathrm{FDR}<0.05)$ enrichment for various neurodevelopmental processes including neurogenesis and a range of cellular GO biological processes: synapse, neuronal part, plasma membrane, cell junction, cytoskeletal, chromosomal and endoplasmic reticulum GO cellular components; protein domain-specific binding GO molecular function; and the axon guidance KEGG pathway (Figure 2b). We examined the extent and timing of expression of candidate genes across brain developmental stages using BrainSpan data in FUMA ${ }^{18}$ and found significant enrichment $(\mathrm{FDR}<0.05)$ for downregulated differentially expressed genes in early infancy (Figure 2c). Expression levels of numerous genes, including $D A A M 1, N T 5 C 2, N E O 1$ recently linked to cortical development, are downregulated during the late pre-natal 26 weeks post-conception) to early post-natal (4 months of age) period (Extended Data Figure 8). These results together suggest that genetic effects on regional brain folding are in part driven via regulation of neuronal development during early brain development. 


\section{Colocalization with brain eQTLs to prioritize candidate genes}

211 We performed colocalization analysis between brain cortical folding loci and the largest

212 cortical eQTL summary dataset generated to date (Metabrain) ${ }^{19}$. We found 27 of 119 loci to be

213 colocalized for at least one sulcal measure with one or more cis eQTLs in the cerebral cortex

214 at a posterior probability (coloc PP4) $>0.7$ and an additional 7 at a suggestive PP4 $>0.5$

215 (Supplementary Table 6, Extended Data Figure 9). A total of 53 unique cortical gene eQTLs

216 colocalized (PP4 >0.7) with at least one sulcal trait in the cortex. 15 of the 27 loci were

217 colocalized with one unique eQTL in the cortex, 9 loci colocalized with 2 eQTLs, 3 with 2

218 eQTLs and the pleiotropic chr17:47Mb MAPT-KANSL1 locus colocalized with 14 different

219 eQTL genes in a complex pattern (Extended Data Figure 9, Supplementary Table 6). Across

220 other brain-related tissues including the cerebellum, basal ganglia, hippocampus and spinal

221 cord, we found a total of 25 loci in the cerebellum, 7 in the basal ganglia, 6 in the hippocampus and 3 in the spinal cord that colocalized (PP4 >0.7) with at least one eQTL, with 9, 2 and 1 colocalized loci in the cerebellum, hippocampus and basal ganglia respectively, not found in cortex tissue.

Multi-trait colocalization of cortex specific $K C N K 2$ eQTL and regional sulcal widths

227 The pleotropic chr1:215Mb locus near $K C N K 2$ is associated with multiple sulcal measures across the brain in a largely symmetrical manner. The strongest lead variant $\sim 40 \mathrm{~Kb}$ upstream of $K C N K 2$, rs1452628:T, exhibited stronger associations with reduced sulcal widths in more superior regions of the brain (Figure 3a, Supplementary Tables 2 and 3). Notably, we observed multiple pairwise colocalizations between significant sulcal width associations at this

232 locus and cortex-specific KCNK2 eQTLs from a large-scale brain tissue eQTL study 233 (MetaBrain) ${ }^{19}$ (Extended Data Figure 9, Supplementary Table 6), where rs1452628:T was 234 associated with increased $K C N K 2$ expression in the cortex only (beta=0.14, $p=8.0 \times 10^{-7}$ ) (cf. 
cerebellum, hippocampus, basal ganglia and spinal cord, all $p>0.1$, Figure $3 \mathbf{b}$ left). We then

236 formally tested whether all or one or more subgroups of the regional sulcal width associations

237 in the locus and cortical KCNK2 eQTL are driven by the same underlying variant using the

238 HyPrColoc multi-trait colocalization approach ${ }^{20}$. We found all associations multi-colocalized

239 to the same variant (posterior probability of colocalization=0.74), with the candidate causal

240 variant, rs1452628, explaining all of the posterior probability of colocalization (Figure 3b

241 right). We further assessed sensitivity to our choice of prior probability of colocalization. Joint

242 colocalization across all or almost all of the traits remained even after sequentially reducing

243 the prior probability (Supplementary Information). These results suggest a shared underlying

244 variant driving all sulcal morphology associations and cortex-specific $K C N K 2$ expression at 245 this locus.

Genetic correlation between brain folding associations and neuropsychiatric conditions

249 Cross referencing with previous non-imaging trait and diseases in the GWAS Catalog, we 250 found that 56 of the 119 loci were associated with one or more diseases or intermediate phenotypes (Supplementary Table 7). We further investigated the genetic correlation (GC) of regional brain folding with 12 neurological diseases, cognitive and psychiatric conditions

(Methods, Supplementary Information). Using an empirical permutation threshold of $p<0.0044$ to account for extensive correlations within brain folding phenotypes and neurorelated illnesses (Methods), we observed 158 significant GCs between regional brain folding measures and 10 neuropsychiatric and cognitive conditions (Supplementary Table 8).

258 Taking the mean GC between each of the four shape parameters and neuropsychiatric conditions, we found at least two distinct clusters, with generalized anxiety disorder (GAD), 
attention deficit hyperactive disorder (ADHD), and major depressive disorder (MDD) and

261 Alzheimer's disease (AD) similarly clustered (Figure 4a). In general, sulcal width measures mostly showed opposite GCs versus the other three sulcal parameters (Figure 4a), in keeping with their correlation structure. Cognitive performance and Parkinson's disease (PD) in particular showed significant positive GCs with length, surface area and mean depth measures across a broad range of brain regions, whilst ADHD and MDD showed negative GCs across those three shape parameters (Supplementary Table 8, Figure 4a and 4b). In particular, we found strongest GCs between PD and central sulcal length $\left(\mathrm{r}_{\mathrm{G}}=0.40, p=3.0 \times 10^{-3}\right)$ and surface area $\left(\mathrm{r}_{\mathrm{G}}=0.33, p=6.0 \times 10^{-4}\right)($ Supplementary Table 8), which indicate the role of sulcal folds adjoining the primary motor cortex in PD. Sulcal width measures mostly showed opposite GCs with neuropsychiatric traits compared the other three sulcal parameters (Figure 4a), in keeping with their correlation structure.

\section{Interactive 3D visualisation of associations}

274 Given the complexity and interdependencies of regional brain folding, visualizing variant association results interactively in $3 \mathrm{D}$ provides more intuitive context to interpret the association results, providing insights into genetic effects across multiple brain regions. We created an interactive resource (https://enigma-brain.org/sulci-browser) where users can query individual genetic variants and visualize the genetic effects across all regional brain folds interactively across all four shape parameters (Figure 5).

281 Visualizing the results, for example, it is clear that pleiotropic associations, such as 
more localised (Figure 5d). We observed strong positive effects of the minor rs4924345:C

286 allele on bilateral central sulcus mean depth $\left(\right.$ beta $\left._{\mathrm{dis}}=0.29, p_{\mathrm{dis}}=3.1 \times 10^{-79}\right)$ and surface area

$287 \quad$ (beta $_{\mathrm{dis}}=0.15, p_{\mathrm{dis}}=6.0 \times 10^{-25}$ ) but negative effects bilaterally on neighbouring superior

288 postcentral intraparietal superior sulcus mean depth $\left(\right.$ beta $\left._{\mathrm{dis}}=-0.14, p_{\mathrm{dis}}=1.0 \times 10^{-18}\right)$ and surface

289 area $\left(\right.$ beta $\left._{\mathrm{dis}}=-0.11, p_{\mathrm{dis}}=6.5 \times 10^{-13}\right)$; retro central transverse ramus of the lateral fissure mean

290 depth ( beta $\left._{\mathrm{dis}}=-0.16, p_{\mathrm{dis}}=7.9 \times 10^{-21}\right)$ and surface area $\left(\right.$ beta $\left._{\mathrm{dis}}=-0.15, p_{\mathrm{dis}}=2.8 \times 10^{-18}\right)$; inferior

291 precentral sulcus mean depth $\left(\right.$ beta $\left._{\mathrm{dis}}=-0.14, p_{\mathrm{dis}}=5.6 \times 10^{-16}\right)$, surface area (beta $\mathrm{dis}=-0.16$,

$\left.292 p_{\text {dis }}=8.0 \times 10^{-19}\right)$ and length ( beta $\left._{\text {dis }}=-0.11, p_{\text {dis }}=1.3 \times 10^{-9}\right)$.

293

294 We have also provided rendering based on effect sizes, Z-scores or $p$-values and an option to 295 download query results. 


\section{Discussion}

297 Cortical gyrification is an orchestrated, multifaceted process that shows striking consistency 298 across individuals ${ }^{21}$. Gyrification is regulated by a complex interplay of cellular, biomechanical and genetic influences ${ }^{9}$ but our understanding of its genetic underpinnings has been limited ${ }^{22,23}$.

300 Abnormalities and variations in brain folding contribute to many common and rare

301 neuropsychiatric conditions. Cortical thickness, surface area and sulcal morphometry are each

302 associated with complex phenotypes such as intelligence ${ }^{24}$, and effects on cortical gyrification are partially independent of those on cortical thickness or surface area ${ }^{25}$.

305 Here, combining densely-imputed genetic variants with whole-exome sequencing, we 306 performed the most comprehensive genetic mapping of regional cortical sulcal morphometry 307 to date, identifying 119 unique genetic loci influencing human sulcal depth, width, length and surface area. We discovered over 60 novel loci not previously implicated in any brain imaging related association studies. The number of genetic associations observed across different sulcal parameters was approximately in accordance with their heritability ${ }^{17}$. We observed stronger genetic correlations than phenotypic correlations between left and right sides, suggesting that

312 environmental and non-genetic factors may play a role in structural and functional

313 lateralization. In particular, regional measures for the most heritable shape parameter, sulcal 314 width, clustered in a way that reflected broad brain topology, indicating that brain sulcal width

315 has a stronger genetic component and is most stable across the lifespan.

317 We demonstrated the highly polygenic genetic architecture of brain folding, which has both

318 local and widespread effects within the brain. When visualised in 3D, local effects are apparent,

319 that are likely to be missed in globally aggregated brain measurement studies. We also 320 implicated specific candidate genes in several cases through coding variants in LD. We added 
321 exonic resolution through WES, as well as through colocalization with brain eQTLs using a

322 large-scale brain specific dataset for better power and specificity ${ }^{19}$. We observed pleiotropic

323 associations at genetic loci consistently implicated in prior genetic studies of neuroimaging

324 phenotypes, such as the MAPT-KANSL1 locus ${ }^{26,27}$, while resolving other associations to

325 specific brain regions and sulcal folding parameters, such as the $K C N K 2$ locus and sulcal width.

326

327 Our results provide evidence of enrichment of associated genes for expression in the cerebral

328 cortex, strongly implicating genes involved in neurodevelopment. We found enrichment for

329 differential gene expression occurring in early brain development, indicating that genetic

330 effects on cortical gyrification occur most prominently during early life, likely via modulation

331 of neurodevelopmental pathways. Inherited functional impairments of these genes and their associated pathways may increase the risk for neurodevelopmental disorders. For example,

333 homozygous and compound heterozygous mutations at EML1 - a gene associated with right

334 insula surface area - cause band heterotopia, a neuronal migration disorder characterized by

335 intellectual disability and epilepsy ${ }^{28}$. Similarly, heterozygous deletion of ZICI and ZIC4 is

336 associated with Dandy-Walker malformation, a congenital cerebellar malformation ${ }^{29}$, whereas

337 contiguous deletions at the 16q24.3 locus encompassing CENPW cause microcephaly,

338 distichiasis, vesico-ureteral and intellectual impairment ${ }^{30}$. Additionally, genetic variants at

339 NUAK1 - a pleiotropic locus associated with frontal, temporal and precentral sulcal widths -

340 have shown links to autism spectrum disorder ${ }^{31,32}, \mathrm{ADHD}^{33}$ and cognitive impairment ${ }^{34}$.

341 Globally, genetic variants influencing cortical gyrification showed robust, widespread

342 correlation with variants influencing cognitive performance, schizophrenia, ADHD and

343 depression, suggesting a shared molecular system potentially underpinning

344 neurodevelopmental and neuropsychiatric disorders ${ }^{35,36}$. 
346 Through multi-trait colocalization, we identified a shared underlying genetic driver of

347 increased cortical $K C N K 2$ expression and pleiotropic effects on reduced sulcal widths. KCNK2,

348 also known as TREK-1, is a two-pore domain potassium channel highly expressed in the

349 central nervous system and modulated by both chemical and physical stimuli. ${ }^{37,38} \mathrm{KCNK} 2$

350 regulates immune-cell trafficking into the $\mathrm{CNS}^{37}$ and genetic ablation of Kcnk2 is associated

351 with neuroinflammation, blood-brain barrier impairment ${ }^{39}$ and increased sensitivity to

352 ischemia and epilepsy in mice ${ }^{40}$. In addition to brain volume, the $K C N K 2$ locus was previously

353 implicated in sulcal opening ${ }^{16}$ and the same lead variant, rs1452628:T, was associated with

354 difference between predicted brain age and chronological age ${ }^{41}$. Our findings re-emphasize the

355 role of $K C N K 2$ in cerebral cortex development, alongside similarly pleiotropic and widely-

356 investigated therapeutic targets such as $N U A K 1^{42}$ and $M A P T^{43}$. Further investigation of the

357 links between these proteins and disease processes downstream of cortical gyrification may

358 support therapeutic development.

360 One notable limitation of the present study is that genetic associations were identified in a population of mostly British individuals. Additionally, dividing UK Biobank participants into discovery and replication cohorts prioritised robustness of genetic associations, but reduced power to detect rare and low frequency variant associations. Larger sample sizes will increase

364 power and refine the estimates reported here. Our method to ascertain brain folding phenotypes

365 is applicable across different MRI scanning protocols, which vary across sites ${ }^{17}$. This should

366 facilitate large-scale, cross-biobank studies of cortical folding and minimise site- and cohortspecific effects.

369 To aid interpretation and increase the utility of our results to the wider scientific community, 370 we created an interactive 3D brain visualisation of our associations, where users can query 
371 specific variant associations across the entire brain and the shape parameters simultaneously.

372 We highlighted various cases where complex and pleiotropic associations differ in brain region

373 and shape parameter distributions, which become more apparent when represented visually in

374 three dimensions.

375

376 In conclusion, we provide the most comprehensive genetic atlas of regional brain folding to

377 date, identifying novel associations and insights into processes that drive the genetic effects, as

378 well as providing a resource for the wider community for further elucidation of specific

379 findings.

380 


\section{Methods}

\section{Samples and participants}

383 UK Biobank (UKB) is a UK population study of approximately 500,000 participants aged 40-

38469 years at recruitment ${ }^{44}$. Participant data include genomic, imaging data, electronic health

385 record linkage, biomarkers, physical and anthropometric measurements. Further details are

386 available at https://biobank.ndph.ox.ac.uk/showcase/. Informed consent were obtained from

387 participants. Analyses in this study were conducted under UK Biobank Approved Project

388 numbers 26041 and 11559.

\section{Brain folding imaging phenotypes}

391 The UK Biobank began collecting brain MRI scans in 2014 with the goal of scanning 100,000

392 individuals. The protocol includes isotropic 3D T1-weighted (T1w) MP-RAGE images (voxel

393 size $1 \mathrm{~mm}^{3}$; field-of-view: $208 \times 256 \times 256$ ) that have undergone bias-field correction in the scanner. Full acquisition details can be found $\mathrm{in}^{45}$. T1w images were processed using FreeSurfer (v7.1.1) (https://surfer.nmr.mgh.harvard.edu/) and quality controlled using protocols developed by the Enhancing Neuro Imaging Genetics for Meta-Analysis (ENIGMA) consortium (http://enigma.ini.usc.edu/). BrainVISA (http://brainvisa.info) was implemented

398 for sulcal classification and labelling ${ }^{46}$. Morphologist 2015, an image-processing pipeline 399 included in BrainVISA, was used to measure sulcal shape descriptors. To improve sulcal extraction and build on current protocols used to analyse thousands of brain scans, quality controlled FreeSurfer outputs (orig.mgz, ribbon.mgz and talairach.auto) were directly

402 imported into the pipeline to avoid re-computing intensities inhomogeneities correction and

403 grey/white matter classification. Sulci were then automatically labelled according to a 404 predefined anatomical nomenclature ${ }^{46,47}$. This protocol is part of the ENIGMA-SULCI 
working group; a Docker and a Singularity container have been created to facilitate the

406 processing

on computational

clusters

407 (https://hub.docker.com/repository/docker/fpizzaga/sulci). We retained length, width, depth, 408 and surface area for all 121 sulcal measurements derived from this protocol for a total of 484 409 phenotypes.

410

411 Phenotypes with missingness $>75 \%$ were excluded from subsequent analysis, leaving 450

412 measurements for analysis. Missingness occurs mainly with smaller sulci that are not identified

413 in some individual MRIs. Prior to analysis, all imaging phenotypes were inverse-rank

414 normalised to approximate a standard normal distribution and minimise effects of outliers. T-

415 distributed stochastic neighbour embedding (t-SNE) was applied on inverse-rank normalised 416 imaging phenotypes.

\section{Discovery and replication cohorts}

419 We partitioned UKB samples with MRI measurements into discovery and replication approximately in 2:1 split. The discovery cohort were comprised of MRI measures in

421 individuals of European ancestry from Newcastle, Stockport and Reading imaging centres,

422 whilst the replication cohort composed of the remaining (non-European) individuals from the 423 aforementioned three centres, and mostly all individuals from the Bristol imaging centre. 424 Subsequent analyses were performed treating the discovery and replication cohorts as 425 completely separate to minimize data contamination and biases.

\section{Genetic data processing}

\section{UKB genetic QC}


UKB genotyping and imputation (and QC) were performed as described previously ${ }^{44}$. WES processed using the RGC SBP pipeline as described $\mathrm{in}^{49,50}$. RGC generated a QC-passing detailed below.

\section{Additional QC and variant processing}

439 In addition to checking for sex mismatch, sex chromosome aneuploidy, and heterozygosity

440 checks, imputed genetic variants were filtered for INFO $>0.8, \mathrm{MAF}>0.01$ (rarer variants around

441 coding regions would be better captured by WES) globally across UKB and chromosome

442 positions were lifted to hg38 build. WES variants were filtered for MAC $>10$ within the UKB

443 subset with MRI measurements. Imputed and WES variants were combined by chromosome position (hg38) and alleles and in the case of overlaps, the WES variant was retained (as WES generally have higher quality calls compared to imputation). Variant annotation was performed using VEP ${ }^{51}$ with Ensembl canonical transcripts used where possible.

\section{Genetic association analyses}

449 GWAS were performed using REGENIE v2.0.1 via a two-step procedure to account for population structure detailed in ${ }^{52}$. In brief, the first step fits a whole genome regression model

451 for individual trait predictions based on genetic data using the leave one chromosome out 452 (LOCO) scheme. We used a set of high-quality genotyped variants: minor allele frequency $453(\mathrm{MAF})>1 \%$, minor allele count $(\mathrm{MAC})>100$, genotyping rate $>99 \%$, Hardy-Weinberg 
equilibrium (HWE) test $p>10^{-15},<10 \%$ missingness and linkage-disequilibrium (LD) pruning

455 (1000 variant windows, 100 sliding windows and $\left.\mathrm{r}^{2}<0.8\right)$. The LOCO phenotypic predictions regression. We limited analyses to variants with $\mathrm{MAC}>50$ to minimise spurious associations.

458 The association models in both steps also included the following covariates: age, age ${ }^{2}$, sex, age* sex, age ${ }^{2 *}$ sex, imaging centre, intracranial volume, first 10 genetic principal components

20 PCs derived from high-quality rare WES variants $(\mathrm{MAF}<1 \%, \mathrm{MAC}>5$, genotyping rate $>99 \%$, HWE test $p>10^{-15},<10 \%$ missingness) as additional control for fine-scale population structure.

\section{Definition and refinement of significant loci}

466 To define significance, we used multiple testing corrected threshold of $p<2 \times 10^{-10}\left(5 \times 10^{-8} / 273\right.$

467 approximate number of independent trait). We used phenotypic PCs accounting for 90\% of

468 phenotype variance to estimate the approximate number of independent traits to account for correlations between regions, side and parameters. Additionally, we also require at least nominal significance $(p<0.05)$ with concordant directions in the replication cohort which should limit false positives even at $p<5 \times 10^{-8}$. For reporting, we also included the standard genome-wide significant loci $\left(p<5 \times 10^{-8}\right)$ that replicated at $p<0.05$ in the replication cohort.

474 We defined independent trait associations through clumping $\pm 500 \mathrm{~Kb}$ around the lead variants using PLINK ${ }^{53}$, excluding the HLA region (chr6:25.5-34.0Mb) which is treated as one locus due to complex and extensive LD patterns. As overlapping genetic regions may be associated

477 with multiple correlated measurements and to avoid over-reporting genetic loci, we merged 
locus.

\section{Cross reference with known genetic associations}

482 We cross-referenced the lead variants and their proxies (LD proxy $\mathrm{r}^{2}>0.8,+/-500 \mathrm{~Kb}$ around 483 the lead variant, with HLA region treated as one region) for significant associations $\left(p<5 \times 10^{-}\right.$

${ }^{8}$ ) in GWAS Catalog . Brain imaging studies were separated from other intermediate and disease phenotypes as defined by the list of brain imaging studies in Supplementary

Information.

487

\section{Expression enrichment}

We examined whether genes within associated loci are enriched for expression the various

brain tissues. Enrichment analysis was performed using the TissueEnrich R package ${ }^{54}$ using

491 the annotated genes (available canonical genes mapped in VEP) for all genome-wide significant variants $\left(p<5 \times 10^{-8}\right.$, additional sensitivity analysis thresholds of $p<5 \times 10^{-7}, 5 \times 10^{-6}$, $5 \times 10^{-5}$ were used for cortex) and a background of annotated genes for all variants analysed. Specifically, we used the RNA dataset from Human Protein Atlas using all genes that are found to be expressed within each tissue.

\section{GO and KEGG process enrichment}

498 Using the same significant annotated genes and backgrounds as for the expression enrichment analyses, we performed enrichment testing for GO and KEGG pathways using the WEB-based

500 GEne SeT AnaLysis Toolkit (WebGestalt) ${ }^{55}$ (http://www.webgestalt.org/). We used the overrepresentation analysis method, analysing GO Biological Process, GO Cellular Component,

502 GO Molecular Function and KEGG, with Benjamini-Hochberg FDR threshold of 0.05 for 503 significance. We used the default parameters of minimum of 5 and maximum 2,000 genes per 
category. Related process and pathway entries were grouped through the inbuilt weighted set cover redundancy reduction approach.

506

\section{FUMA analyses of expression timing}

508 Gene expression enrichment across BrainSpan (https://www.brainspan.org/) brain ages and 509 developmental stages was analysed based on averaged $\log 2$ transformed expression levels

510 across each label. Genes were defined as differentially expressed when the Bonferroni

511 corrected $p<0.05$ and the absolute log fold change $\geq 0.58$ between specific brain ages or

512 developmental stages compared to others ${ }^{18}$. All other annotated genes/transcripts in the

513 BrainSpan data were included as background genes for comparison in hypergeometric tests of 514 gene sets. Significantly enriched gene sets had FDR corrected $p<0.05$.

\section{Genetic correlation analysis}

517 We performed genetic correlation analysis between brain folding phenotypes (including

518 hemispheres and shape parameters), and 12 neuropsychiatric conditions with readily available 519 summary data using LD score regression (LDSC v1.0.1) ${ }^{56}$. We also performed SNP-based 520 heritability estimation using LDSC. Genetic variants were filtered and processed using the

521 "munge_sumstats.py" in LDSC and we used LD scores recommended by the software 522 authors ${ }^{56}$.

524 To account for multiple testing of extensive related and correlated phenotypes, we permuted 525 each neuropsychiatric condition Z-score 100 times (limited by computational cost) and tested 526 each permuted neuropsychiatric condition with each brain folding phenotype to generate an 527 empirical multiple testing threshold of $p=0.0044$ (approximately adjusted $p<0.01$ from 100 528 permutations). 


\section{Colocalization analyses}

531 We performed colocalization analyses ${ }^{57}$ between brain eQTLs from MetaBrain and brain

532 folding loci using the coloc R package. We used the default priors ( $\mathrm{p} 1=10^{-4}, \mathrm{p} 2=10^{-4}, \mathrm{p} 12^{-}=10^{-}$

$533{ }^{5}$ ) with regions defined as $+/-500 \mathrm{~Kb}$ around the lead variant. Evidence for colocalization was

534 assessed using the posterior probability (PP) for hypothesis 4 (PP4; an association for both

535 traits driven by the same causal variant). PP4 $>0.5$ were deemed likely to colocalize as it

536 guaranteed that hypothesis 4 was computed to have the highest posterior probability, and

537 PP4 $>0.7$ were deemed highly likely to colocalize.

539 To assess whether all traits jointly colocalize at the $K C N K 2$ locus we used the multi-trait 540 colocalization software $\mathrm{HyPrColoc}{ }^{20}$, using the recommended default settings and priors

541 (HyPrColoc's default prior parameters $\mathrm{p}=10^{-4}$ and $\mathrm{p}_{\mathrm{c}}=2 \times 10^{-2}$ are equivalent to setting $\mathrm{p} 1=10^{-4}$,

$542 \mathrm{p} 2=10^{-4}, \mathrm{p} 12=2 \times 10^{-6}$ in coloc, hence the default prior probability of colocalization $\mathrm{p} 12$ is

543 slightly more conservative than in coloc). HyPrColoc computes evidence supporting one or

544 more clusters of traits colocalizing at a single variant in the region, concluding that a cluster of

545 traits colocalize if the posterior probability of colocalization (PPC) is above a user defined

546 threshold ( $\mathrm{PPC}>0.5$ by default, which is equivalent to setting the algorithms' regional, $\mathrm{P}_{\mathrm{R}}$, and

547 alignment, $\mathrm{P}_{\mathrm{A}}$, thresholds to 0.7 respectively). We also performed additional sensitivity 548 analysis across different parameter specifications (Supplementary Information). 


\section{Acknowledgements}

551 We thank all the participants, contributors and researchers of UK Biobank for making data

552 available for this study. We thank the UK Biobank Exome Sequencing Consortium (AbbVie,

553 Alnylam Pharmaceuticals, AstraZeneca, Biogen, Bristol-Myers Squibb, Pfizer, Regeneron and

554 Takeda) for generation the whole exome sequencing data and Regeneron Genetics Centre for

555 initial quality control of the exome sequencing data. We thank Peter Kochunov and his team

556 for hosting the interactive browser, with support from NIH instrumentation grant

557 S10OD023696. N.J. is supported by NIH grant R01AG059874. S.E.M. and J.N.P. are supported in part by NHMRC grants APP1172917 and APP1158127.

560 Data availability

561 The online browser for visualisation of results is available at https://enigma-brain.org/sulci-

562 browser.

\section{Code availability}

564 Codes used are part of standard software and tools. Additional details available in Methods.

\section{Author contributions}

567 Study conceptualization and design: C.D.W., P.M.T., N.J., B.B.S.; methodology: B.B.S., S.J.L.,

568 P.M.T., N.J., C.D.W.; sulcal imaging processing: F.P., A.Z., D.D., T.I., I.B.G., N.J.; phenotype

569 harmonisation: M.J., D.G.M., S.S.C., Biogen Biobank Team; analysis: B.B.S., S.J.L., J.N.P.,

570 S.E.M., C.N.F.; interactive browser: N.S., F.P.; writing: B.B.S., C.D.W., P.M.T., N.J., H.R.;

571 all authors critically reviewed the manuscript. 
574 The authors declare the following competing interests: B.B.S., S.J.L., Biogen Biobank Team,

575 M.J., D.G.M., H.R., C.D.W. are employees of Biogen. P.M.T and N.J received grant support

576 from Biogen for this work.

577

578 Correspondence

579 Correspondence and requests for materials should be addressed to B.B.S., N.J., P.M.T. or

580 C.D.W.

581 
bioRxiv preprint doi: https//doi.org/10.1101/2021.10.13.463489; this version posted October 15,2021 . The copyright holder for this preprint (which was not certified by peer review) is the author/funder, who has granted bioRxiv a license to display the preprint in perpetuity. It is made available under aCC-BY-NC-ND 4.0 International license.

\section{Figures}

583 Figure 1. Summary of brain sulcal association results. (a) Schematic of brain sulcal folds and

584 shape parameters. Brain region legend corresponds to colours in figures a-c. (b) t-SNE of regional 585 brain sulcal measures for each shape parameter. (c) GWAS association results by shape 586 parameters and side. Diamonds indicate lead (sentinel) associations that replicated $(p<0.05)$. Points 587 above 0 in the $y$-axis in each plot refers to associations with left sided sulcal measures, below 0 with 588 right sided measures. Diamonds along 0 is the y-axis indicate lead associations for bilateral sulcal 589 measures. Dashed horizonal line indicate GWAS significance threshold $\left(p=5 \times 10^{-8}\right)$. (d) Summary of 590 number of associations by side and shape parameters. (e) Top: genetic and phenotypic correlation 591 between left and right sides. Middle: Genetic correlation between shape parameters. Bottom: 592 Phenotypic correlation between shape parameters. Middle and bottom: left hemisphere correlations in upper triangle, right hemisphere correlations in lower triangle.

594
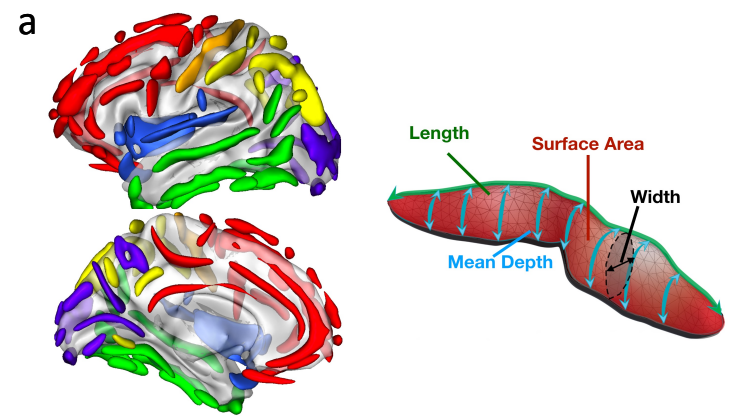

b

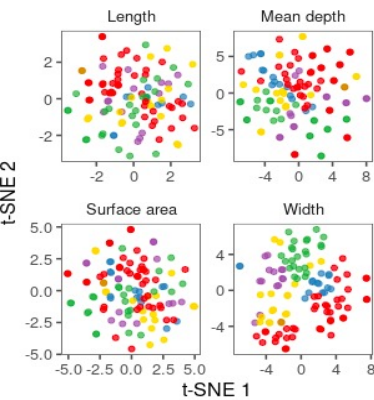

C

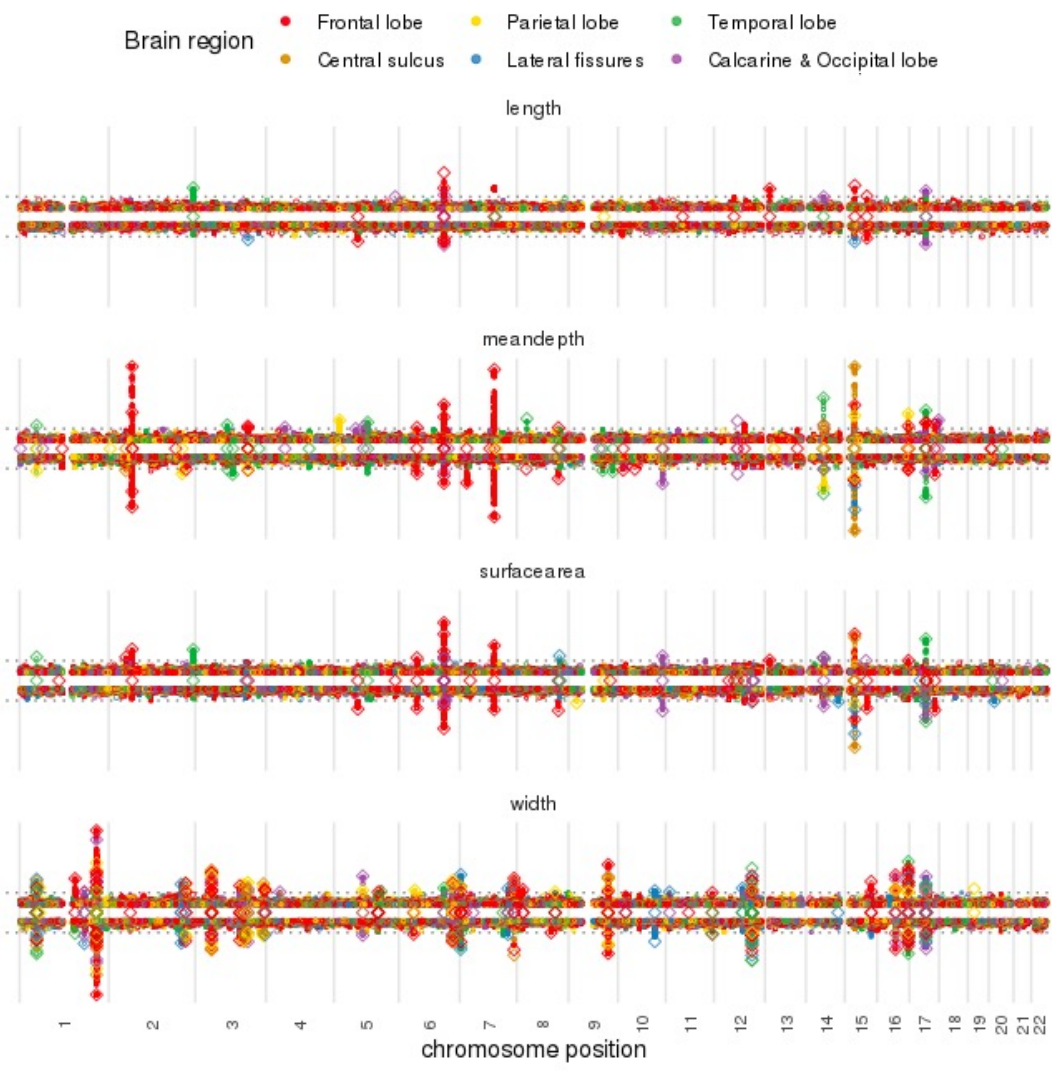

\begin{tabular}{l|c|c|c}
\hline & Left & Right & Bilateral \\
\hline Length & $10(5)$ & $9(2)$ & $17(9)$ \\
Mean depth & $32(15)$ & $39(17)$ & $70(35)$ \\
Surface area & $23(9)$ & $28(15)$ & $54(23)$ \\
Width & $127(62)$ & $120(61)$ & $194(95)$ \\
Total & $192(91)$ & $196(95)$ & $335(162)$ \\
\hline
\end{tabular}

e
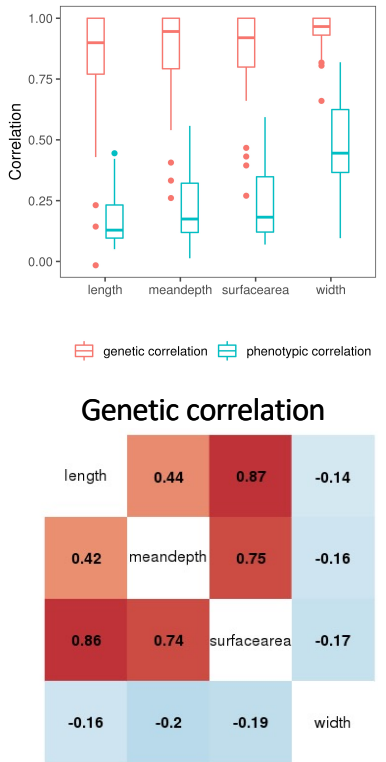

Phenotypic correlation

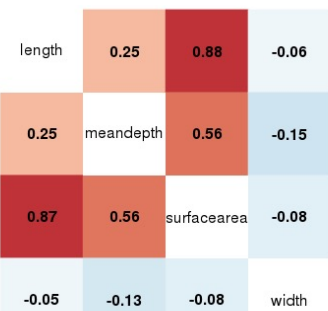


bioRxiv preprint doi: https://doi.org/10.1101/2021.10.13.463489; this version posted October 15, 2021. The copyright holder for this preprint (which was not certified by peer review) is the author/funder, who has granted bioRxiv a license to display the preprint in perpetuity. It is made available under aCC-BY-NC-ND 4.0 International license.

597 Figure 2. Enrichment of genes in significant loci for: (a) gene expression across various tissues 598 (inset shows sensitivity analysis at other GWAS thresholds), (b) GO and KEGG pathways $599 \quad(\mathrm{FDR}<0.05)$, (c) differentially expressed genes across brain development stages.

600

a

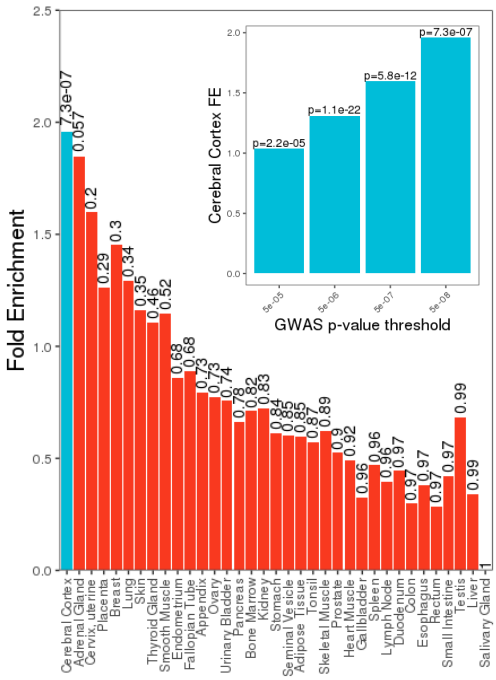

b

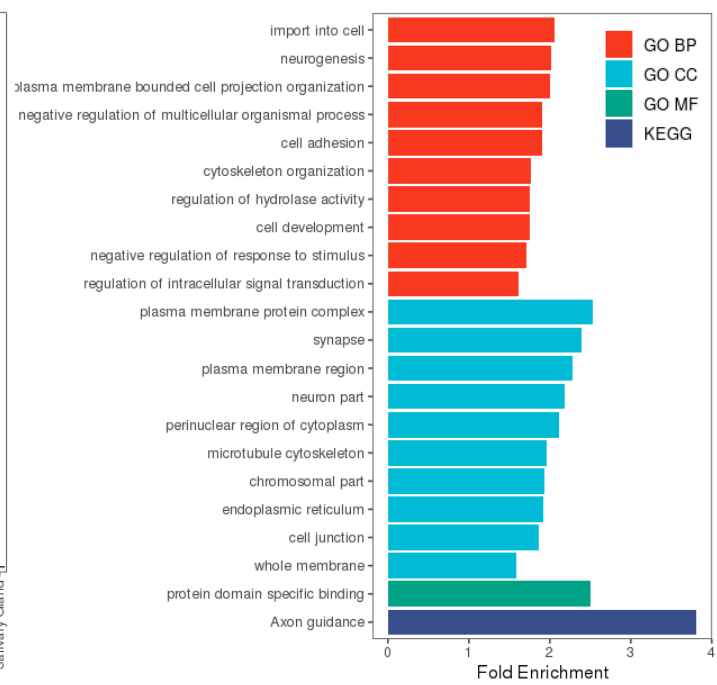

C

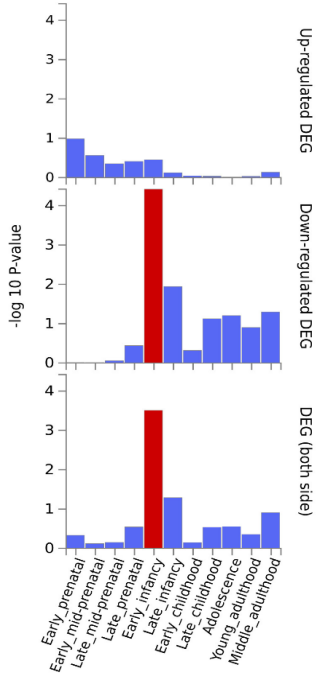

602 
Figure 3. KCNK2 locus associations. (a) Association of the lead rs1452628: T variant with reduced 604 sulcal widths across the brain. (Grey colours indicate associations with $p_{\text {rep }}>0.05$ ). (b) Left: regional association plot of MetaBrain KCNK2 eQTLs for spinal cord, basal ganglia, hippocampus and cerebellum. Right: regional association plots and colocalization of cortex $K C N K 2$ eQTL and different lead variants in the KCNK2 locus. A subset of associations shown for each different lead

608 variant shown due to space constraints.

609

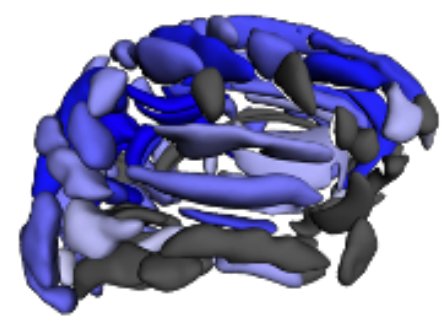

b

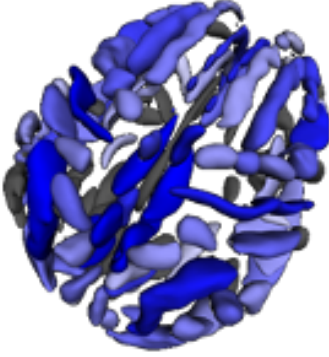

Spinal Cord eQTL
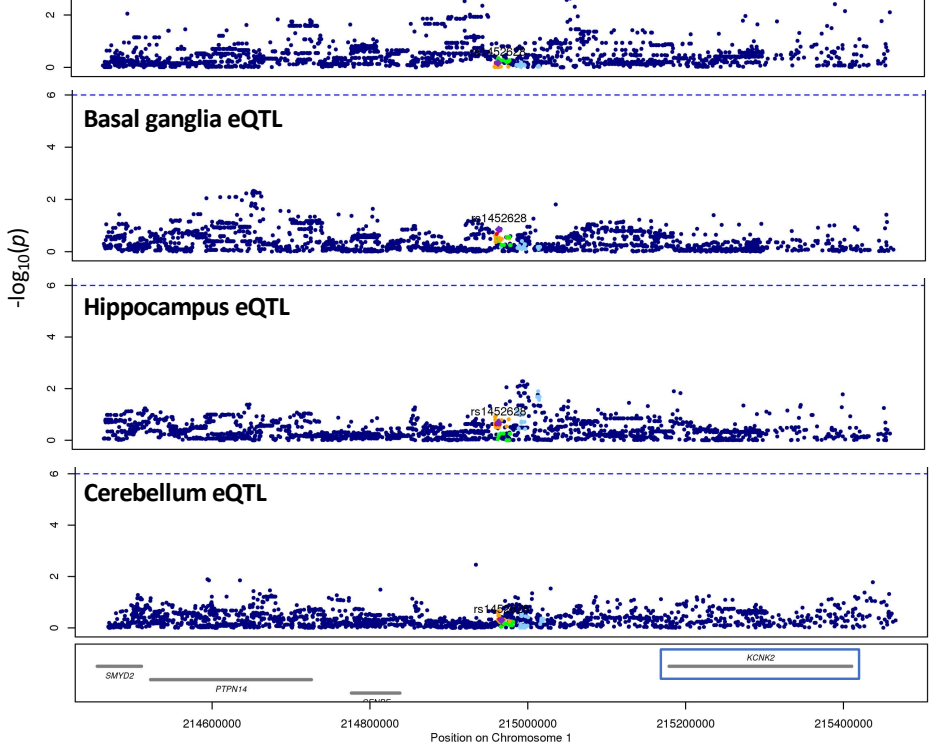

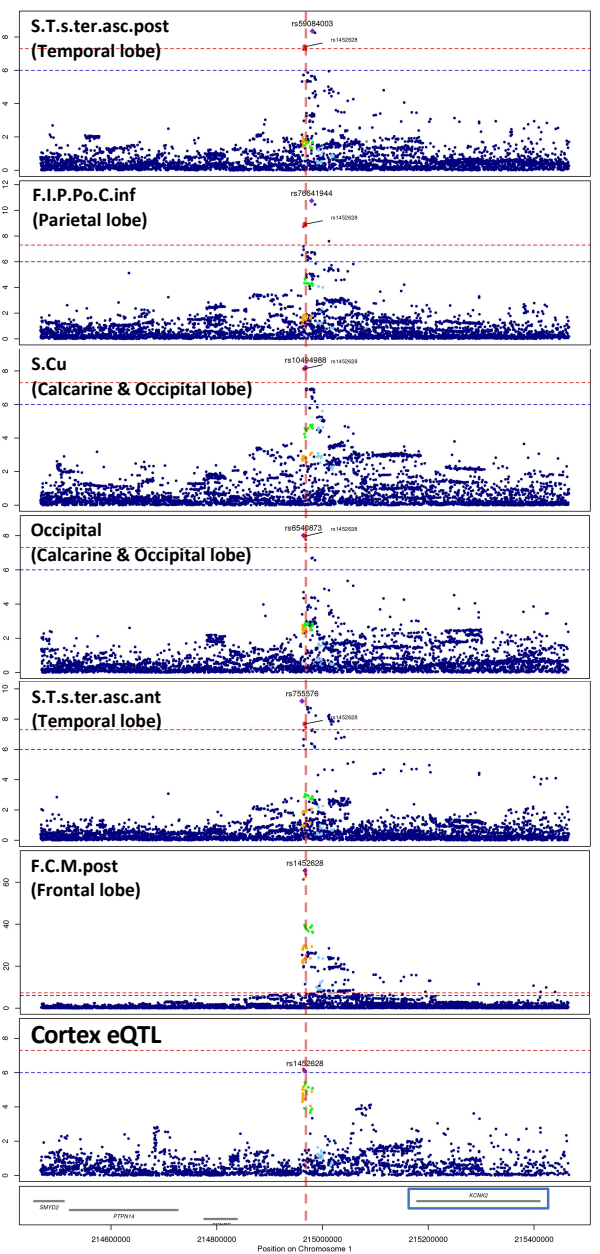

610

611

612 
613 Figure 4. (a) Genetic correlations between shape parameters and neuropsychiatric conditions. (b)

614 Examples of genetic correlations across brain sulcal folds with cognitive performance,

615 Parkinson's disease, attention deficit hyperactive disorder (ADHD) and major depressive 616 disorder.

617

a

\begin{tabular}{|c|c|c|c|c|c|c|c|c|c|c|c|c|}
\hline 0.04 & 0.06 & 0.01 & 0.03 & -0.02 & -0.01 & -0.03 & -0.01 & -0.01 & 0.07 & 0.01 & -0.02 & width \\
\hline 0.00 & -0.07 & -0.01 & -0.03 & 0.13 & 0.04 & 0.05 & 0.05 & 0.06 & 0.02 & 0.02 & 0.02 & mean depth \\
\hline-0.16 & -0.10 & -0.06 & -0.08 & 0.14 & 0.08 & 0.03 & 0.04 & 0.03 & 0.01 & 0.01 & -0.02 & length \\
\hline .07 & -0.10 & -0.05 & -0.06 & 0.15 & 0.08 & 0.04 & 0.05 & 0.05 & 0.02 & 0.02 & 0.00 & surface area \\
\hline
\end{tabular}

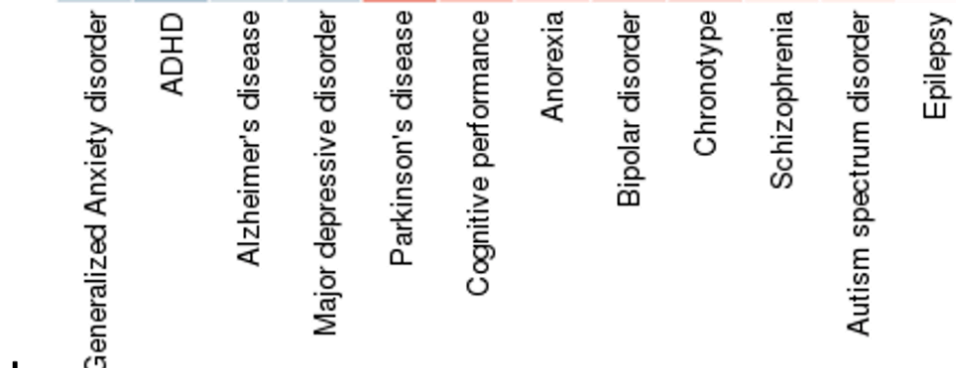

b Cognitive performance Parkinson's disease

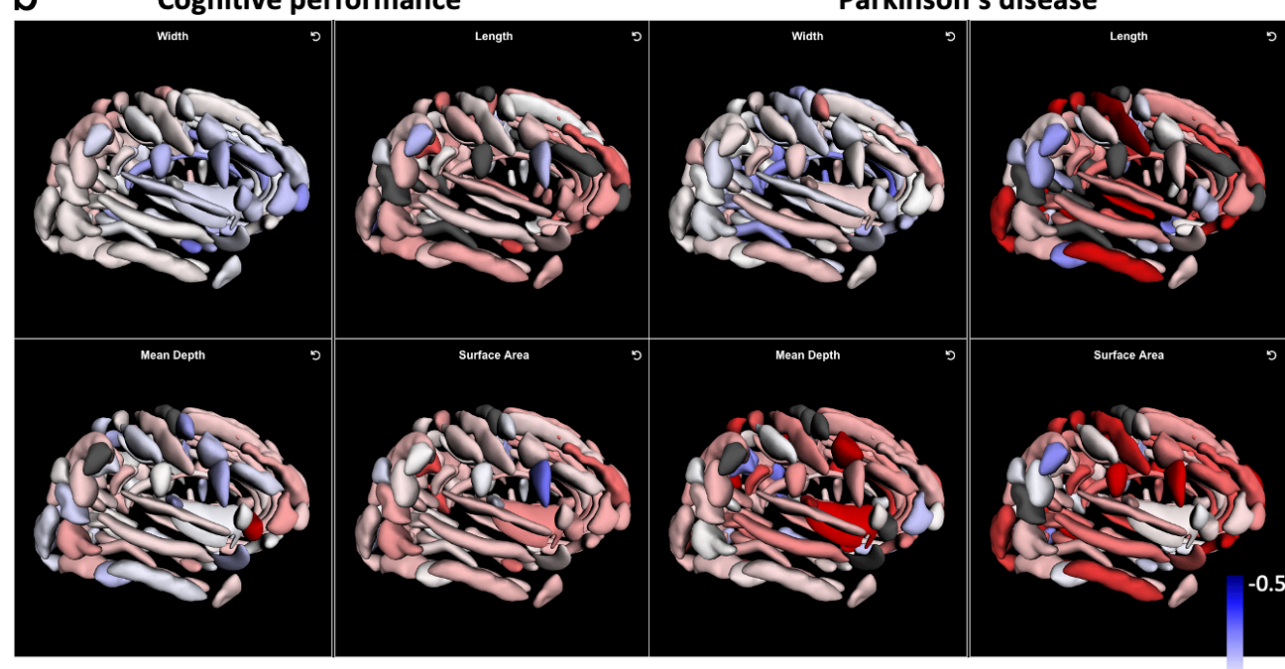

ADHD

Major depressive disorder

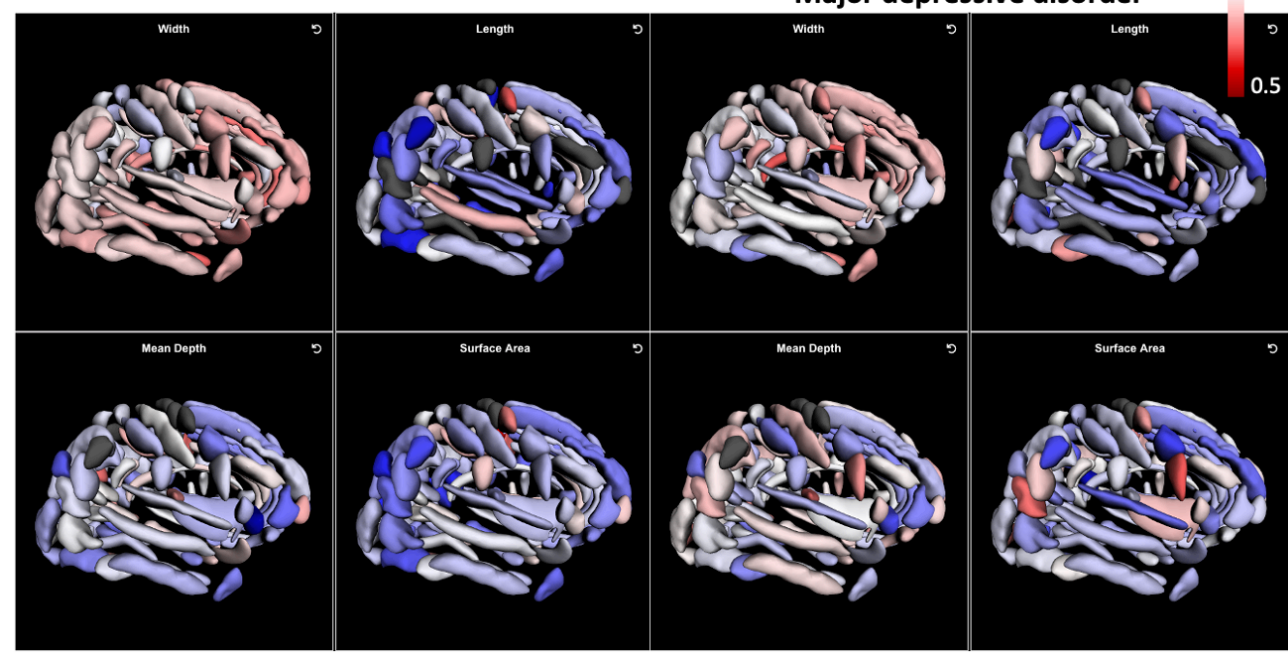


619 Figure 5. Three-dimensional visualisation of brain sulcal associations (Z-scores) for four 620 exemplar pleiotropic loci.

a

rs12146713 - chr12:106Mb NUAK1
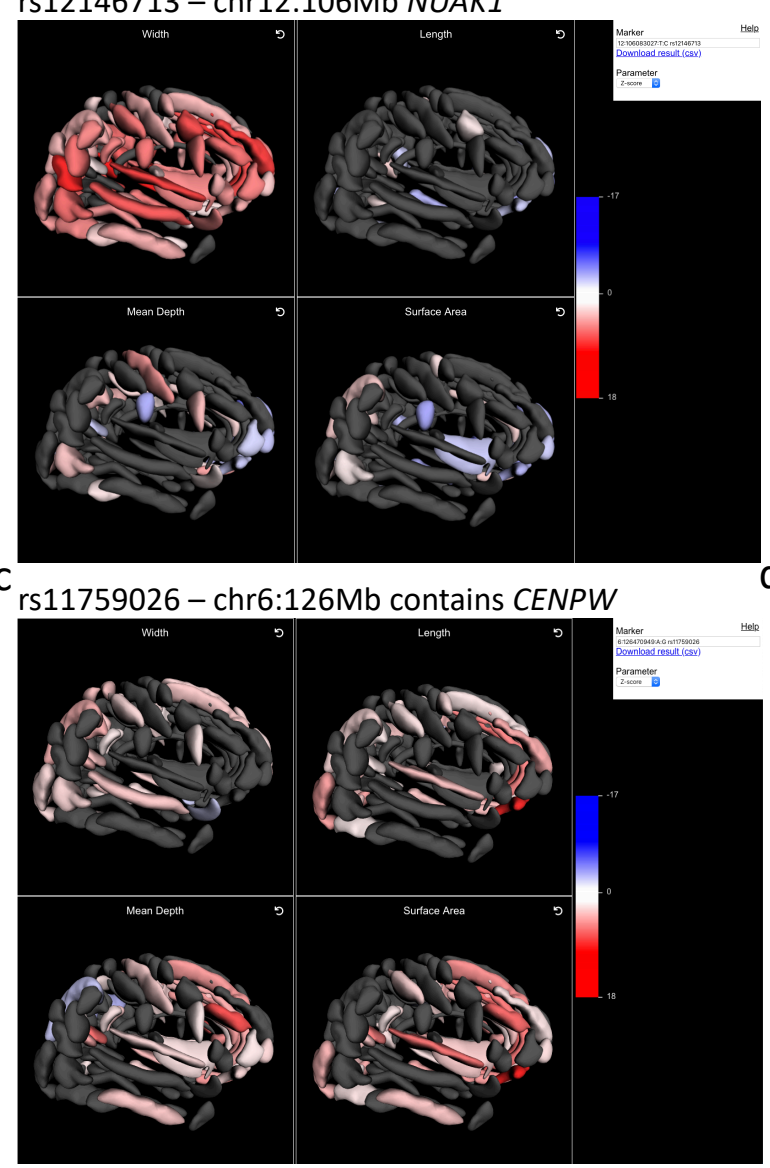

b rs4843553 - chr16:87Mb C16orf95

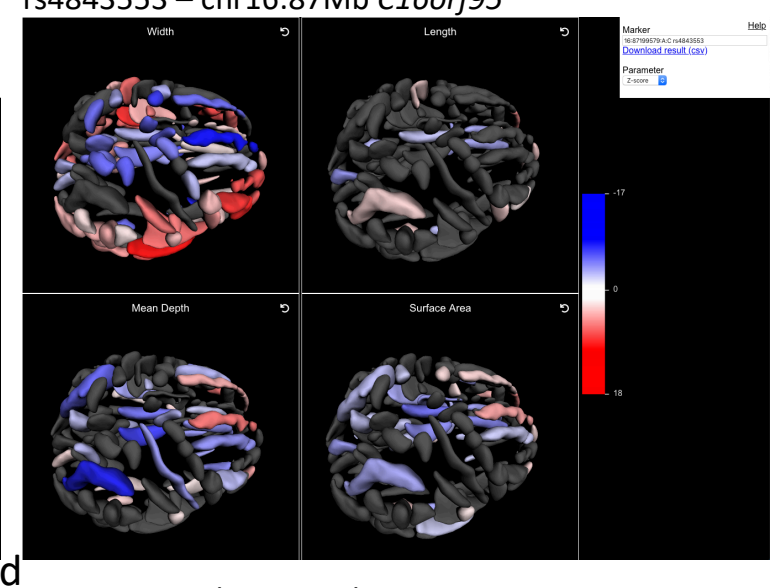

rs2033939 - chr15:39Mb Intergenic

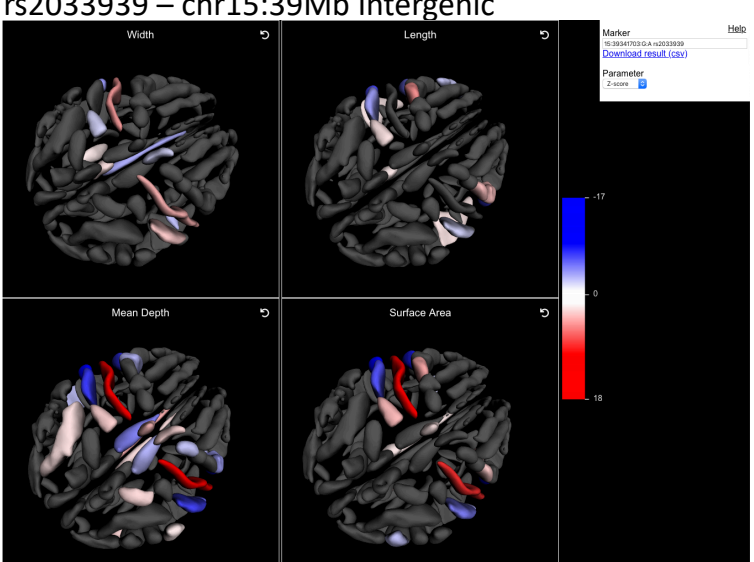

621

622 


\section{References}

6251 Hibar, D. P. et al. Common genetic variants influence human subcortical brain 626 structures. Nature 520, 224-229, doi:10.1038/nature14101 (2015).

6272 Elliott, L. T. et al. Genome-wide association studies of brain imaging phenotypes in 628 UK Biobank. Nature 562, 210-216, doi:10.1038/s41586-018-0571-7 (2018).

6293 Shen, L. \& Thompson, P. M. Brain Imaging Genomics: Integrated Analysis and Machine Learning. Proc IEEE Inst Electr Electron Eng 108, 125-162, doi:10.1109/JPROC.2019.2947272 (2020).

4 Lee, P. H. et al. Partitioning heritability analysis reveals a shared genetic basis of brain anatomy and schizophrenia. Mol Psychiatry 21, 1680-1689, doi:10.1038/mp.2016.164 (2016).

5 Hofer, E. et al. Genetic correlations and genome-wide associations of cortical structure in general population samples of 22,824 adults. Nat Commun 11, 4796, doi:10.1038/s41467-020-18367-y (2020).

6 Buniello, A. et al. The NHGRI-EBI GWAS Catalog of published genome-wide association studies, targeted arrays and summary statistics 2019. Nucleic acids research 47, D1005-D1012, doi:10.1093/nar/gky1120 (2019).

7 Sasabayashi, D., Takahashi, T., Takayanagi, Y. \& Suzuki, M. Anomalous brain gyrification patterns in major psychiatric disorders: a systematic review and transdiagnostic integration. Translational Psychiatry 11, 176, doi:10.1038/s41398021-01297-8 (2021).

8 Ronan, L. \& Fletcher, P. C. From genes to folds: a review of cortical gyrification theory. Brain Struct Funct 220, 2475-2483, doi:10.1007/s00429-014-0961-z (2015).

9 Llinares-Benadero, C. \& Borrell, V. Deconstructing cortical folding: genetic, cellular and mechanical determinants. Nat Rev Neurosci 20, 161-176, doi:10.1038/s41583018-0112-2 (2019).

10 Besson, P., Andermann, F., Dubeau, F. \& Bernasconi, A. Small focal cortical dysplasia lesions are located at the bottom of a deep sulcus. Brain 131, 3246-3255, doi:10.1093/brain/awn224 (2008).

11 Hong, S. E. et al. Autosomal recessive lissencephaly with cerebellar hypoplasia is associated with human RELN mutations. Nat Genet 26, 93-96, doi:10.1038/79246 (2000).

12 DeTure, M. A. \& Dickson, D. W. The neuropathological diagnosis of Alzheimer's disease. Mol Neurodegener 14, 32, doi:10.1186/s13024-019-0333-5 (2019).

13 Hamelin, L. et al. Sulcal morphology as a new imaging marker for the diagnosis of early onset Alzheimer's disease. Neurobiol Aging 36, 2932-2939, doi:10.1016/j.neurobiolaging.2015.04.019 (2015).

14 Cai, K. et al. Identification of Early-Stage Alzheimer's Disease Using Sulcal Morphology and Other Common Neuroimaging Indices. PLoS One 12, e0170875, doi:10.1371/journal.pone.0170875 (2017).

15 van der Meer, D. et al. The genetic architecture of human cortical folding. bioRxiv, 2021.2001.2013.426555, doi:10.1101/2021.01.13.426555 (2021).

16 Le Guen, Y. et al. eQTL of KCNK2 regionally influences the brain sulcal widening: evidence from 15,597 UK Biobank participants with neuroimaging data. Brain Struct Funct 224, 847-857, doi:10.1007/s00429-018-1808-9 (2019).

17 Pizzagalli, F. et al. The reliability and heritability of cortical folds and their genetic correlations across hemispheres. Commun Biol 3, 510, doi:10.1038/s42003-020- 
18 Watanabe, K., Taskesen, E., van Bochoven, A. \& Posthuma, D. Functional mapping and annotation of genetic associations with FUMA. Nat Commun 8, 1826, doi:10.1038/s41467-017-01261-5 (2017).

19 de Klein, N. et al. Brain expression quantitative trait locus and network analysis reveals downstream effects and putative drivers for brain-related diseases. bioRxiv, 2021.2003.2001.433439, doi:10.1101/2021.03.01.433439 (2021).

20 Foley, C. N. et al. A fast and efficient colocalization algorithm for identifying shared genetic risk factors across multiple traits. Nat Commun 12, 764, doi:10.1038/s41467020-20885-8 (2021).

21 Garcia, K. E., Kroenke, C. D. \& Bayly, P. V. Mechanics of cortical folding: stress, growth and stability. Philos Trans R Soc Lond B Biol Sci 373, doi:10.1098/rstb.2017.0321 (2018).

22 Van Essen, D. C. A 2020 view of tension-based cortical morphogenesis. Proc Natl Acad Sci U S A, doi:10.1073/pnas.2016830117 (2020).

23 Van Essen, D. C. A tension-based theory of morphogenesis and compact wiring in the central nervous system. Nature 385, 313-318, doi:10.1038/385313a0 (1997).

24 Tadayon, E., Pascual-Leone, A. \& Santarnecchi, E. Differential Contribution of Cortical Thickness, Surface Area, and Gyrification to Fluid and Crystallized Intelligence. Cereb Cortex 30, 215-225, doi:10.1093/cercor/bhz082 (2020).

25 Panizzon, M. S. et al. Distinct genetic influences on cortical surface area and cortical thickness. Cereb Cortex 19, 2728-2735, doi:10.1093/cercor/bhp026 (2009).

26 Adams, H. H. et al. Novel genetic loci underlying human intracranial volume identified through genome-wide association. Nat Neurosci 19, 1569-1582, doi:10.1038/nn.4398 (2016).

27 Chu, S. A. et al. Brain volumetric deficits in MAPT mutation carriers: a multisite study. Ann Clin Transl Neurol 8, 95-110, doi:10.1002/acn3.51249 (2021).

28 Kielar, M. et al. Mutations in Eml1 lead to ectopic progenitors and neuronal heterotopia in mouse and human. Nat Neurosci 17, 923-933, doi:10.1038/nn.3729 (2014).

29 Grinberg, I. et al. Heterozygous deletion of the linked genes ZIC1 and ZIC4 is involved in Dandy-Walker malformation. Nat Genet 36, 1053-1055, doi:10.1038/ng1420 (2004).

30 Butler, M. G. et al. Microcephaly, intellectual impairment, bilateral vesicoureteral reflux, distichiasis, and glomuvenous malformations associated with a 16q24.3 contiguous gene deletion and a Glomulin mutation. Am J Med Genet A 158A, 839849, doi:10.1002/ajmg.a.35229 (2012).

31 Courchet, V. et al. Haploinsufficiency of autism spectrum disorder candidate gene NUAK1 impairs cortical development and behavior in mice. Nat Commun 9, 4289, doi:10.1038/s41467-018-06584-5 (2018).

32 Iossifov, I. et al. De novo gene disruptions in children on the autistic spectrum. Neuron 74, 285-299, doi:10.1016/j.neuron.2012.04.009 (2012).

33 Alemany, S. et al. New suggestive genetic loci and biological pathways for attention function in adult attention-deficit/hyperactivity disorder. Am J Med Genet B Neuropsychiatr Genet 168, 459-470, doi:10.1002/ajmg.b.32341 (2015).

34 Johnson, M. R. et al. Systems genetics identifies a convergent gene network for cognition and neurodevelopmental disease. Nat Neurosci 19, 223-232, doi:10.1038/nn.4205 (2016).

35 Cristino, A. S. et al. Neurodevelopmental and neuropsychiatric disorders represent an interconnected molecular system. Mol Psychiatry 19, 294-301, doi:10.1038/mp.2013.16 (2014). 
36 Jensen, M. \& Girirajan, S. Mapping a shared genetic basis for neurodevelopmental disorders. Genome Med 9, 109, doi:10.1186/s13073-017-0503-4 (2017).

37 Bittner, S. et al. Endothelial TWIK-related potassium channel-1 (TREK1) regulates immune-cell trafficking into the CNS. Nat Med 19, 1161-1165, doi:10.1038/nm.3303 (2013).

38 Djillani, A., Mazella, J., Heurteaux, C. \& Borsotto, M. Role of TREK-1 in Health and Disease, Focus on the Central Nervous System. Front Pharmacol 10, 379, doi:10.3389/fphar.2019.00379 (2019).

39 Fang, Y. et al. Deficiency of TREK-1 potassium channel exacerbates blood-brain barrier damage and neuroinflammation after intracerebral hemorrhage in mice. $J$ Neuroinflammation 16, 96, doi:10.1186/s12974-019-1485-5 (2019).

40 Heurteaux, C. et al. TREK-1, a K+ channel involved in neuroprotection and general anesthesia. EMBO J 23, 2684-2695, doi:10.1038/sj.emboj.7600234 (2004).

41 Jonsson, B. A. et al. Brain age prediction using deep learning uncovers associated sequence variants. Nat Commun 10, 5409, doi:10.1038/s41467-019-13163-9 (2019).

42 Lasagna-Reeves, C. A. et al. Reduction of Nuak1 Decreases Tau and Reverses Phenotypes in a Tauopathy Mouse Model. Neuron 92, 407-418, doi:10.1016/j.neuron.2016.09.022 (2016).

43 Congdon, E. E. \& Sigurdsson, E. M. Tau-targeting therapies for Alzheimer disease. Nat Rev Neurol 14, 399-415, doi:10.1038/s41582-018-0013-z (2018).

44 Bycroft, C. et al. The UK Biobank resource with deep phenotyping and genomic data. Nature 562, 203-209, doi:10.1038/s41586-018-0579-z (2018).

45 Miller, K. L. et al. Multimodal population brain imaging in the UK Biobank prospective epidemiological study. Nat Neurosci 19, 1523-1536, doi:10.1038/nn.4393 (2016).

46 Riviere, D. et al. Automatic recognition of cortical sulci of the human brain using a congregation of neural networks. Med Image Anal 6, 77-92, doi:10.1016/s13618415(02)00052-x (2002).

47 Mangin, J. F. et al. Object-based morphometry of the cerebral cortex. IEEE Trans Med Imaging 23, 968-982, doi:10.1109/TMI.2004.831204 (2004).

48 Szustakowski, J. D. et al. Advancing human genetics research and drug discovery through exome sequencing of the UK Biobank. Nature Genetics 53, 942-948, doi:10.1038/s41588-021-00885-0 (2021).

49 Van Hout, C. V. et al. Exome sequencing and characterization of 49,960 individuals in the UK Biobank. Nature 586, 749-756, doi:10.1038/s41586-020-2853-0 (2020).

50 Kosmicki, J. A. et al. A catalog of associations between rare coding variants and COVID-19 outcomes. medRxiv, 2020.2010.2028.20221804, doi:10.1101/2020.10.28.20221804 (2021).

51 McLaren, W. et al. The Ensembl Variant Effect Predictor. Genome Biol 17, 122, doi:10.1186/s13059-016-0974-4 (2016).

52 Mbatchou, J. et al. Computationally efficient whole-genome regression for quantitative and binary traits. Nat Genet 53, 1097-1103, doi:10.1038/s41588-02100870-7 (2021).

53 Chang, C. C. et al. Second-generation PLINK: rising to the challenge of larger and richer datasets. Gigascience 4, 7, doi:10.1186/s13742-015-0047-8 (2015).

54 Jain, A. \& Tuteja, G. TissueEnrich: Tissue-specific gene enrichment analysis. Bioinformatics 35, 1966-1967, doi:10.1093/bioinformatics/bty890 (2019).

55 Liao, Y., Wang, J., Jaehnig, E. J., Shi, Z. \& Zhang, B. WebGestalt 2019: gene set analysis toolkit with revamped UIs and APIs. Nucleic Acids Res 47, W199-W205, doi:10.1093/nar/gkz401 (2019). 
56 Bulik-Sullivan, B. K. et al. LD Score regression distinguishes confounding from polygenicity in genome-wide association studies. Nat Genet 47, 291-295, doi:10.1038/ng.3211 (2015).

776

57 Giambartolomei, C. et al. Bayesian test for colocalisation between pairs of genetic association studies using summary statistics. PLoS Genet 10, e1004383, doi:10.1371/journal.pgen.1004383 (2014). 